\section{Tool not a discipline}

Enzyme Kinetics: Behaviour and Analysis of Rapid Equilibrium and Steady-State Enzyme Systems. By Irwin H. Segal. Pp xxii+957. (WileyInterscience: New York and London, July 1975.) $£ 15.00$.

ONE might have expected a book on enzyme kinetics by I. H. Segal to make as useful a contribution to its field as his Biochemical Calculations has made within its own category of elementary textbooks. Unfortunately, this has proved otherwise. His new volume is a long and detailed account of just one aspect of enzyme kinetics: steady state methods. Yet it is possible to read through its excess of 900 pages and at the end of it not to have learnt anything about the behaviour of enzymes. The rationale of the study of the kinetics of enzyme reactions is surely the information that kinetic methods provide about the operation of enzymes, either from the viewpoint of the elucidation of the chemical mechanism of the enzyme or the understanding of some biological phenomena. In my opinion, enzyme kinetics is a tool of biochemical research, not an intellectual discipline within its own right. This book presents the latter view.

The heart of the book is a catalogue of steady-state rate equations for enzyme mechanism containing one, two or three substrates reacting with the enzyme in all possible sequences together with the inhibition patterns expected for different types of inhibitors. The mathematics have been presented clearly and double-reciprocal plots of reaction velocity against reactant concentration illustrate most of the equations. No examples of the use of these equations in enzymology are given, which is regrettable because the value of a method can only be judged by the problems it solves. The nomenclature of enzyme mechanisms used throughout the book is that of Cleland. But the principles of steady state kinetics were established more than 20 years ago by Haldane, Alberty and Dalzeil, who demonstrated when these methods could distinguish between alternative reaction mechanisms and, equally important when they could not. The newer nomenclature of Cleland is useful on account of its systematic approach but yields no new principles nor unifying concepts. The same comment might apply to this book. The effort here has been expended on description rather than comprehension. For instance, it has never seemed to me helpful to use terms such as Bi Uni Uni Uni Ping Pong Ter Bi to denote a reaction mechanism since they fail to advance an understanding of the mechanism at the physical level.
Therefore, this book is recommended only to professionals working in the field of steady state enzyme kinetics. Though the derivation of the rate equations for any single mechanism is tedious rather than difficult, some will find it useful to have available a compendium of ssteady state equations for a variety of different systems derived for them. But the restriction to a single experimental method treated in a purely theoretical manner renders the book unsuitable for the general student of biochemistry. One can admire the rigour with which the author approaches his subject but not the basic premise from which he starts.

S. E. Halford

\section{Breath of air}

Principles of Comparative Respiratory Physiology. By Pierre Dejours. Pp. xvi +253. (North Holland: Amsterdam and Oxford; American Elsevier: New York, 1975.) Dff. 35; $\$ 14.75$.

HaviNG an almost exclusively neurophysiological interest in respiratory novements, I undertook the reviewing of Principles of Respiratory Physiology with some trepidation, since I knew this would force me to tread very unfamiliar paths through the jungle of work on respiratory gas exchange in different animals. I am glad to say that my fears were unfounded. True Frenchman that he is, Dr Dejours has created a masterful 'Guide Bleu' through this jungle, providing a clear trail for the timorous, yet offering the more adventuresome rich local detail and source references on which to linger.

I particularly like the way in which he deals with the relevant physical principles of diffusional and convective gas flows, the $\mathrm{O}_{2}$ and $\mathrm{CO}_{2}$ capacitances of the gaseous and fluid compartments of the respiratory system and their corresponding $\mathrm{O}_{2}$ and $\mathrm{CO}_{2}$ conductances. The operation of these principles in the living organism is illustrated throughout the book by appropriate reference to phylogenetic or species specific mechanisms of gas exchange in both water and air breathers representing the most lowly and most complex of animals. The net result of this endeavour is that Dejours has produced an impressive, overall view of gas exchange even for the most complex Metazoa in which he traces the flow of $O$. from the environment to the mitachondrion by way of the alveolar gas, gas-blood barrier, blood, systemic capillary walls, interstitial fluids and cell membrane, together with the flow of $\mathrm{CO}$, in the reverse direction.

The clear authority of the author is no better marked than in the closing chapter where he expresses the view point that the principal aim in the control of respiration is the adequate oxygenation of the tissues in contrast to the more commonly held view that it is the $\mathrm{CO}_{z}$ pressure and acid-base balance which are primarily regulated. His defence rests on the evidence that only long term changes in the needs of oxygenation lead to significant adaptive morphological, biochemical and physiological changes of the organism.

Highly recommended to all air breathers interested in respiration.

T. A. Sears

\section{Electronic structure}

Electron Densities in Molecules and Molecular Orbitals. (Physical Chemistry: Series of Monographs, Volume 35.) By John R. Van Wazer and Ilyas Absar. Pp. ix +101 . (Academic: New York and London, September 1975.) $\$ 14.50 ; £ 7.25$.

ThIS is an unusual book. Essentially the authors have a computer programme for Hartree Fock electronic wave function calculations for molecules incorporating flexible possibilities for the basis sets and a perspective three-dimensional graphic output expressing the electron density throughout a cross-sectional plane. This they use for total and for one-electron densities for $\mathrm{LiF}, \mathrm{H}_{2} \mathrm{O}, \mathrm{HCP}, \mathrm{C}_{2} \mathrm{H}_{6}$, $\mathrm{PF}_{3} \mathrm{O}$ and some 30 other molecules. The book largely presents these graphics and associate text for undergraduate chemists "whose minds are spatially oriented"; for the more expert there is little except possible illustrations for a lecture.

A greater attention to detail is necessary, such as clear bold labelling of the molecule for each figure, clear indications of the nuclear positions, clearer depiction of nodal lines, omission of slapdash jargon--such as reference to the $C_{3}$ axis of $\mathrm{PHF}_{2} \mathrm{O}$ and careful spatial orientation of all figures relating to the same molecule such as 3.1 and 3.2 on page 29 . Emphasis on the shape of the electron density in a single cross section downgrades the importance of kinetic energy, ignores spin effects, ignores time correlation features associated with configuration interaction and leaves the weaker student with a dominant picture of molecular orbitals as sharp peaks on each nucleus (where $\psi^{2}$ remains large for $s$ orbitals) surrounded by a trough (associated with the relatively unimportant spherical nodal surface in $2 \mathrm{~s}$ orbitals) and a few small bumps associated with bonds.

Others may agree that there are better ways of introducing simple electronic structure to undergraduates.

D. H. Whiffen 